\title{
Efecto del estrés por sombra sobre la producción en plantas de limonio (Limonium sp. cv. Bluestream)
}

\author{
Effect of stress by shading on sea lavender \\ (Limonium sp. cv. Bluestream) plants yield
}

FANOR CASIERRA-POSADA ${ }^{1}$

DEIVY L. MORENO²
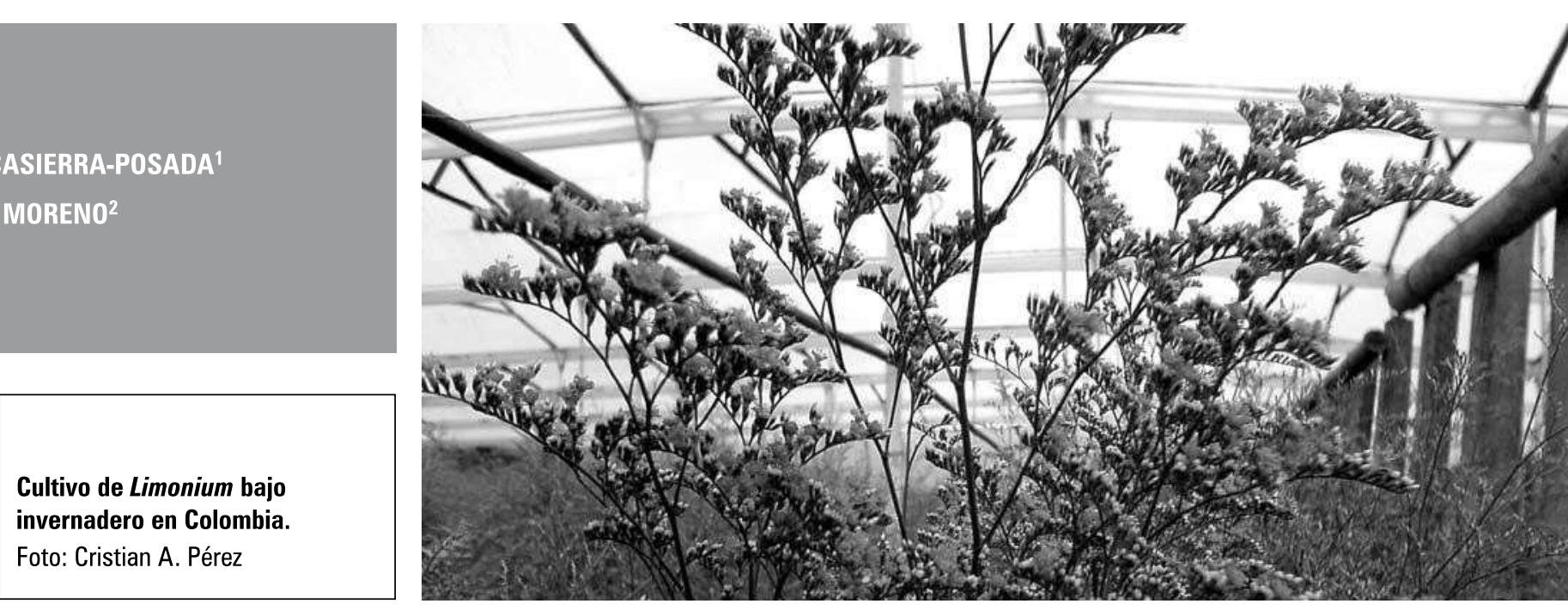

\section{RESUMEN}

Bajo condiciones naturales, la luz ambiental disponible para las plantas varía espacial y temporalmente en términos de calidad, cantidad y duración. La reducción en la cantidad de luz afecta numerosos aspectos del desarrollo vegetal. Muchos de esos efectos están relacionados con la reducción en la fotosíntesis, con sus consecuentes cambios en la disponibilidad de azucares. Las respuestas más estudiadas al respecto son la inducción de la floración y la elongación de tallos y pecíolos. Se evaluó el efecto de la sombra antes del corte de las flores en plantas de limonio cv. Bluestream sobre el número de hojas y de flores cosechadas, el peso específico de las hojas y la longitud del tallo de las flores. Las plantas se desarrollaron bajo invernadero en Guasca (Colombia). Para inducir el sombreado, se desplegaron mallas polisombra de 47 y 80\% de reducción de luz, 1,5 m por encima de las plantas. Las plantas control crecieron sin red por encima. Los resultados mostraron reducción, mediante la red, en todos los parámetros evaluados, en comparación con las plantas control, pero la longitud del tallo de la flor fue influenciada negativamente en mayor grado por la sombra. Los tallos florales fueron 36,5 y 49,0\% más cortos que aquellos cosechados las plantas control bajo las mallas de 47 y $80 \%$ de reducción de luz, respectivamente, afectando severamente la calidad de la flor para exportación. Los resultados sugieren una alta sensibilidad de las plantas de limonio a la reducción de la luz.

\footnotetext{
Profesor Asociado, Grupo de Investigación Ecofisiología Vegetal, Facultad de Ciencias Agropecuarias, Universidad Pedagógica y Tecnológica de Colombia, Tunja (Colombia). fanor.casierra@uptc.edu.co

2 Ingeniero Agrónomo, Universidad Pedagógica y Tecnológica de Colombia, Tunja (Colombia). deivyleo@yahoo.com
} 
Palabras clave adicionales: flores de corte, calidad de flor, tolerancia al estrés.

\section{ABSTRACT}

Under natural conditions, the environmental light of plants varies spatially and temporally in terms of quality, quantity and duration. Reduction in light quantity affects numerous aspects of plant development. Many of these effects are related to the reduction in photosynthesis and consequential changes in sugar availability. The most studied responses are those of the induction of flowering and the elongation of stems and petioles. The effect of shading prior to cut of flowers in sea lavender plants $\mathrm{cv}$. Blue stream on number of leaves and harvested flowers, the leaf specific weight and the flower stem length were evaluated. Plants grew under greenhouse conditions in Guasca (Colombia). For shading, black nets of 47 and $80 \%$ light reduction $1.5 \mathrm{~m}$ over plants were deployed. Control plants grew without a net over. Results showed reduction by all evaluated parameters through nets in comparison to control plants, but the stem length was more negatively influenced by shading. Flower stems were 36.5 and $49.0 \%$ shorter in comparison to those harvested in control plants under nets of 47 and $80 \%$ of light reduction, respectively, affecting strongly the flower quality for exportation. Results suggested high sensibility of sea lavender plants for light reduction.

Additional key words: cut flowers, flower quality, stress tolerance.

Fecha de recepción: 04-09-2007

Aprobado para publicación: 28-11-2007

INTRODUCCIÓN

Limonium es un tipo de planta que produce una flor de corte utilizada en la elaboración de bouquets, en los que se combinan especies de colores variados, lo que otorga al ramo un valor agregado, razón por la cual las compañías productoras de flores cultivan diferentes especies de este género con miras a lograr un mayor contraste en el colorido del bouquet (Chamorro et al., 2007). $86 \%$ de la producción de flores de corte en Colombia proviene de la Sabana de Bogotá (Asocolflores, 2004), de zonas con alta nubosidad, causal de reducción en la radiación incidente, a pesar de que se reporta que los representantes de este género son muy exigentes en luz (Escher, 1996). Estos factores determinan que las plantas se deben adaptar a las condiciones de baja iluminación para cumplir con sus funciones metabó- licas y mantener una producción adecuada, con base en su posible plasticidad fenotípica.

La plasticidad fenotípica es la capacidad de un organismo de producir fenotipos diferentes, en respuesta a cambios en el ambiente (Schmalhausen, 1949); es así como las plantas pueden manifestar ajustes en sus características morfológicas o fisiológicas en respuesta a una variación en la disponibilidad de recursos. Estos ajustes facilitan la aclimatación de las plantas a condiciones ambientales cambiantes o contrastantes (Schlichting, 1986), con lo que se consigue un incremento en su capacidad competitiva. Se han detectado respuestas plásticas en diversas especies vegetales, tanto intolerantes como de tolerancia intermedia (Bazzaz y Carlson, 1982; Abrams y 
Mosteller, 1995). No obstante, la capacidad de aclimatación de las plantas varía en función de su tolerancia (Lambers et al., 1998) y al mismo tiempo de acuerdo a la magnitud de limitación del recurso (Guarnaschelli et al., 2004). Para predecir los posibles efectos de control de la vegetación natural en cultivos, es importante conocer las respuestas de crecimiento de las plantas en campo y su capacidad de aclimatación ante diferentes niveles de déficit de recursos.

En diversos trabajos se analizan las respuestas de las plantas frente a condiciones de estrés por uno o varios factores ambientales (Tognetti et al., 1994; Abrams y Mosteller, 1995; Alexieva et al., 2003; Castro-Díez et al., 2006). Se conoce que si las plantas de Lycopersicon esculentum se iluminan continuamente, se desarrolla una clorosis fuerte y se inhibe la floración, lo que sugiere que la luz es uno de los factores más importantes en la regulación de la diferenciación floral en esta especie (Kristoffersen, 1963); por el contrario en Capsicum annuum, la sombra induce incremento en la caída de flores, con su respectiva consecuencia negativa sobre el cuajado de frutos (Shifriss et al., 1994).

En Malus domestica se encontró que la sombra redujo la tasa de transpiración y la temperatura alrededor de las hojas (Casierra-Posada y Lüdders, 2001), disminuyó el valor de los parámetros determinantes de la calidad de fruta (Casierra-Posada, 1999), pero no afectó el contenido de minerales en hojas y brotes (Casierra-Posada y Lüdders, 2001a). Del mismo modo, una reducción de la luz del orden de $33 \%$ redujo la fotosíntesis neta en horas de la mañana en Malus domestica en días muy iluminados en comparación con árboles a plena exposición; además, la temperatura de la hoja se redujo por la sombra en valores cercanos a $3^{\circ} \mathrm{C}$; sin embargo, a partir de las 11:00 a.m., la tasa fotosintética fue ligeramente superior en plantas sombreadas que en plantas a plena exposición, lo que sugiere que la disminución esperada en cuanto a la fotosíntesis neta por la reducción de la luz, fue compensada con una disminución en la tasa respiratoria (Ebert y Casierra, 2000).

En términos de crecimiento de la hoja, se ha encontrado que la reducción en la cantidad de luz puede ser causal tanto de la reducción como del incremento en la expansión foliar (Wilson, 1966; Dengler, 1980; Volenec y Nelson, 1982; Lichtentahler, 1983; Schnyder y Nelson, 1988). El sombreado de las plantas causa con frecuencia reducción en la temperatura de las hojas, lo que podría tener un impacto sobre el crecimiento y expansión foliar (Ben Haj Salah y Tardieu, 1995; Granier y Tardieu, 1998; Lafarge et al., 1998; Granier et al., 2002).

Quizá el factor más estudiado bajo condiciones de sombreado es su efecto sobre la inducción de la floración (Franklin y Whitelam, 2005) y sobre la elongación de tallos y pecíolos (Kozuka et al., 2005). Adicionalmente, el sombreado induce cambios en la morfología foliar y alteraciones en el desarrollo de las hojas, a nivel celular (Dengler, 1980; Lee et al., 2000; Yano y Terashima, 2004).

El propósito del presente estudio fue la evaluación de la producción y calidad de la flor en plantas de limonio 'Bluestream' expuestas a diferentes niveles de sombreado, bajo invernadero, en un cultivo comercial, con miras a establecer la tolerancia de la especie a condiciones de iluminación disminuida.

\section{MATERIALES Y MÉTODOS}

El ensayo se desarrolló en la vereda Santa Ana, del municipio de Guasca, Cundinamarca, en la finca C.I. Fase Flores Ltda., cuya ubicación coresponde a $4^{\circ} 52^{\prime}$ latitud $\mathrm{N}$ y $73^{\circ} 53^{\prime}$ longitud $\mathrm{O}$, a una altura de $2.656 \mathrm{msnm}$. Como material vegetal se utilizaron plantas de Limonium sp. 'Bluestream'. El estudio se realizó en camas de $30 \mathrm{~m}^{2}$, en las cuales crecieron las plantas a una densidad de siembra de 4 plantas por $\mathrm{m}^{2}$ y un número de 30 plantas por tratamiento. 
Para el montaje del ensayo, se utilizó malla polisombra de 47 y $80 \%$ de reducción de luz, las cuales dejaban pasar 24.320 y 13.542 lux (lx) a las plantas, medidos con un luxómetro 0500 (Lux-meter, Testoterm $\mathrm{GmbH} \&$ Co. Alemania). Estas intensidades luminosas correspondieron a una reducción en la luminosidad en el orden de 63,8 y $79,8 \%$ respectivamente, en relación con la luminosidad determinada fuera del invernadero. Dentro del invernadero, se registró una intensidad luminosa de 44.062 lx, que correspondió al tratamiento control. Por tanto, el invernadero restó $34,4 \%$ de la iluminación incidente, medida a plena exposición.

El experimento consistió en un ensayo factorial, con tres niveles de iluminación, con diseño completamente al azar y seis replicaciones, tomando cinco plantas como unidad experimental. La información se analizó mediante un análisis de variancia y la prueba de separación de promedios de Tukey $(P<0,01)$ con el programa SPSS versión 11.5.1 (Statistical Product and Service Solutions).

\section{RESULTADOS Y DISCUSIÓN}

Se encontró que la reducción de la luz en términos de 47 y $80 \%$ indujo disminución del número de hojas en valores de 15,9 y $17,3 \%$, respectivamente, con respecto al número de hojas desarrolladas en las plantas control. Los resultados para esta variable arrojaron diferencia altamente significativa $(P<0,01)$ en cuanto a los dos niveles de sombreamiento en relación con el control, pero no hubo diferencia entre los tratamientos en donde se colocó sombra a las plantas (figura 1).

Las variables relacionadas con el área para la captura de luz, tales como área foliar y número de hojas se vieron afectadas por el sombreado, lo cual está relacionado con la tolerancia de las plantas a este factor causante de estrés. Al respecto, Kitagima (1994) y Walters y Reich (1999) rechazaron la predicción de Givnish (1988); quien afirmaba que la tolerancia a la sombra se basaría en la maximización de la captura neta de carbono bajo condiciones de luz baja, tras encontrar que las plántulas jóvenes de las especies tolerantes desarrollaron una razón de área foliar más baja que especies pioneras, mientras que la información obtenida de etapas ontogénicas más avanzadas sí brindan algo de apoyo a esta propuesta, ya que las plantas juveniles de las especies tolerantes a veces despliegan una gran superficie asimilatoria (King, 1994; Niinemets 1998; Lusk y Contreras 1999). Esta aparente contradicción se resuelve considerando las consecuencias a largo

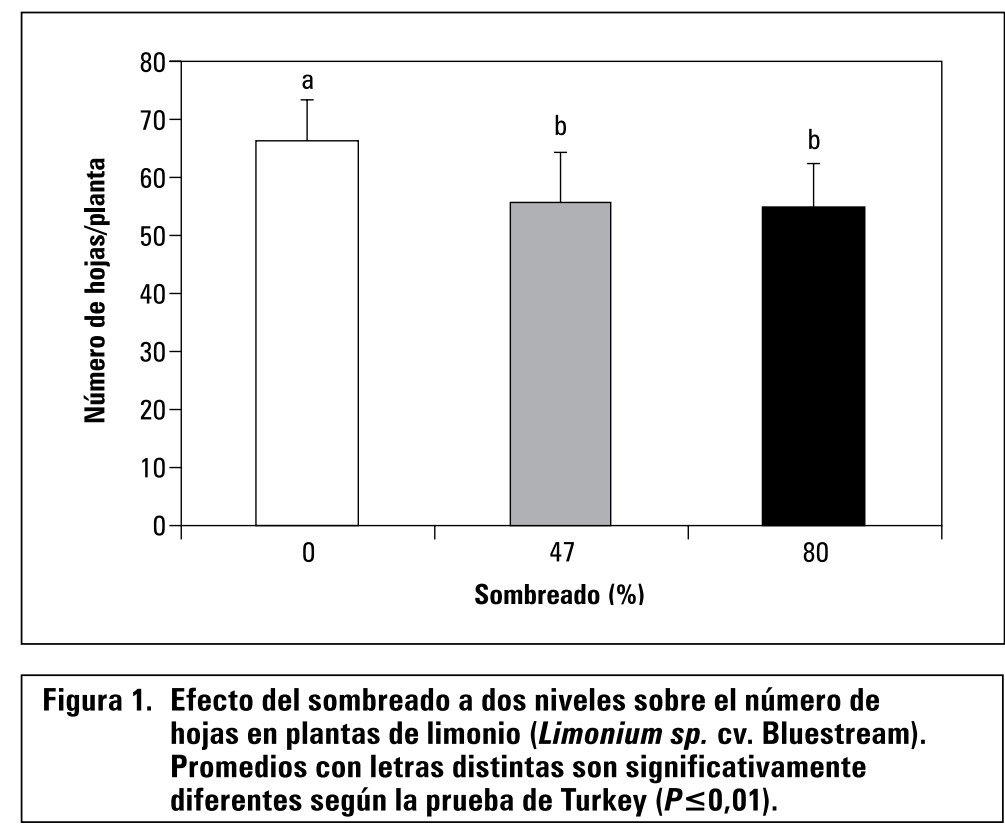


plazo de la variación interespecífia en la longevidad foliar (Lusk, 2002). A pesar de que plántulas de las especies tolerantes siempreverdes inician su ciclo de vida con una menor razón de área foliar (cociente entre el área foliar total y el peso seco total de la planta) que las especies pioneras, estas primeras acumulan varias cohortes de hojas mientras el recambio de follaje suele ser bastante rápido en las últimas. Por ende, aunque las especies pioneras anualmente asignan una mayor proporción de su producción a nuevas hojas cuando crecen a la sombra, dentro de unos pocos años de crecimiento lento en estos ambientes su razón de área foliar decae a valores inferiores a los presentados por las especies tolerantes.

En consecuencia, la tolerancia a la sombra en especies siempreverdes parece resultar, por lo menos parcialmente, de una combinación de rasgos que maximizan la captura neta de energía en luz baja a largo plazo, pero no la tasa de crecimiento (Lusk, 2002). La estrategia a largo plazo de las especies tolerantes mediante la conformación de hojas duraderas, permite la acumulación de una gran superficie foliar a pesar de los modestos aumentos anuales determinados por la escasez de luz. No obstante, la ventaja en captura neta de energía indicada por la distribución de biomasa en plantas juveniles, las tasas de crecimiento de las especies tolerantes a la sombra, en general, son semejantes o inferiores a las de las pioneras (Kitagima, 1994; Veneklaas y Poorter, 1998; Walters y Reich 1999; Lusk 2002). Esta discrepancia se correlaciona con las diferencias en la asimilación de fotoasimilados en donde las especies tolerantes almacenan más que las intolerantes (Kobe, 1997).

Como consecuencia de la sombra el peso específico de las hojas se redujo. Es así como las mallas polisombra de 47 y $80 \%$ de disminución de la luz provocaron una reducción de 17,4 y 22,9\% en el valor de esta variable, en relación con el presentado por las plantas control. El análisis de variancia de esta información mostró diferencias altamente significativas $(P<0,01)$, como se presenta en la figura 2.

En cuanto a la densidad de las hojas, se reporta que en los hábitat productivos, la competencia apremia las altas tasas de crecimiento, que se posibilitan con la producción de hojas de construcción liviana de alto rendimiento fotosintético por unidad de biomasa. Sin embargo, este tipo de hoja es desventajoso en aquellos hábitats donde las condiciones adversas, como la sombra,

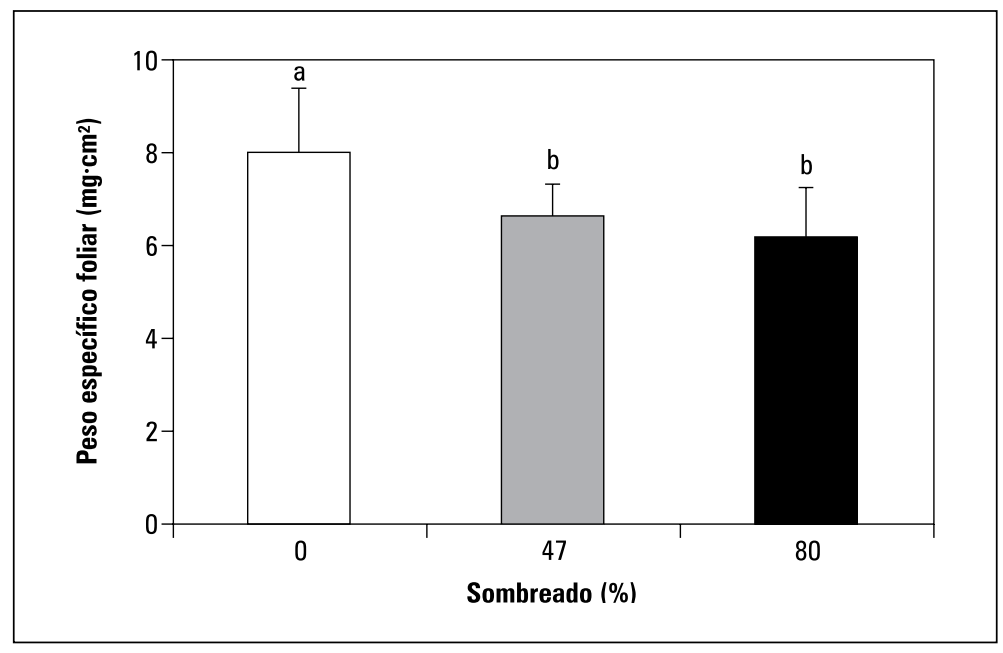

Figura 2. Efecto del sombreado a dos niveles sobre el peso específico de las hojas en plantas de limonio (Limonium sp. cv. Bluestream). Promedios con letras distintas son significativamente diferentes según la prueba de Turkey $(P \leq 0,01)$. 
imposibilitan el despliegue de todo su potencial. En los hábitats crónicamente adversos, donde la conservación de los recursos es clave para la supervivencia, es más relevante una hoja robusta que puede mantener un desempeño modesto durante varias temporadas (Lambers y Poorter 1992; Reich 1993; Lusk 2004).

Las hojas de las especies sombratolerantes suelen ser más gruesas y densas que las de especies pioneras (Kitajima 1994, Walters y Reich 1999), permitiendo una vida activa de varios años (Williams et al.; 1989; King, 1994). En contraste, un recambio rápido de hojas delgadas es común en las especies intolerantes, por lo menos en las especies pioneras de vida corta que logran altas tasas de crecimiento. Con estos argumentos, se pone de manifiesto la sensibilidad de las plantas de limonio a la sombra, pues esta induce la producción de hojas de baja densidad, propias de especies intolerantes a la sombra.

La cantidad de tallos cosechados luego del sombreado disminuyó con los tratamientos impuestos a las plantas. En aquellas que crecieron bajo las mallas de 47 y $80 \%$ de reducción de la luz se pudieron cosechar respectivamente 25,5 y $29,4 \%$ tallos menos que los cosechados en plantas con- trol. En cuanto a esta variable se presentó diferencia altamente significativa $(P<0,01)$ entre los tratamientos evaluados (figura 3 ).

La longitud promedio del tallo de las flores fue severamente afectada por la sombra. Es así como plantas expuestas a sombreado de 47 y $80 \%$ produjeron respectivamente tallos 36,5 y 49,0\% más cortos en relación con los producidos por las plantas de control. El análisis de variancia de esta información mostró diferencias altamente significativas $(P<0,01)$, como se presenta en la figura 4 .

Bajo condiciones de sombreado hubiera sido de esperarse la producción de tallos de mayor longitud, como resultado de la morfogénesis orientada por la sombra, mediante la cual, las plantas expuestas a condiciones disminuidas de iluminación producen tallos de mayor longitud, pero más delgados que aquellos desarrollados en condiciones normales de iluminación, como sucede con Anemone coronaria, en donde la producción de flores decae cuando se cultivan a plena exposición, pero bajo condiciones de sombreado (55 y $67 \%$ de sombra), producen plantas más altas sin variaciones en el rendimiento de las flores. Además, se recomienda por tanto el uso de sombra en condiciones de alta luminosidad en diferentes

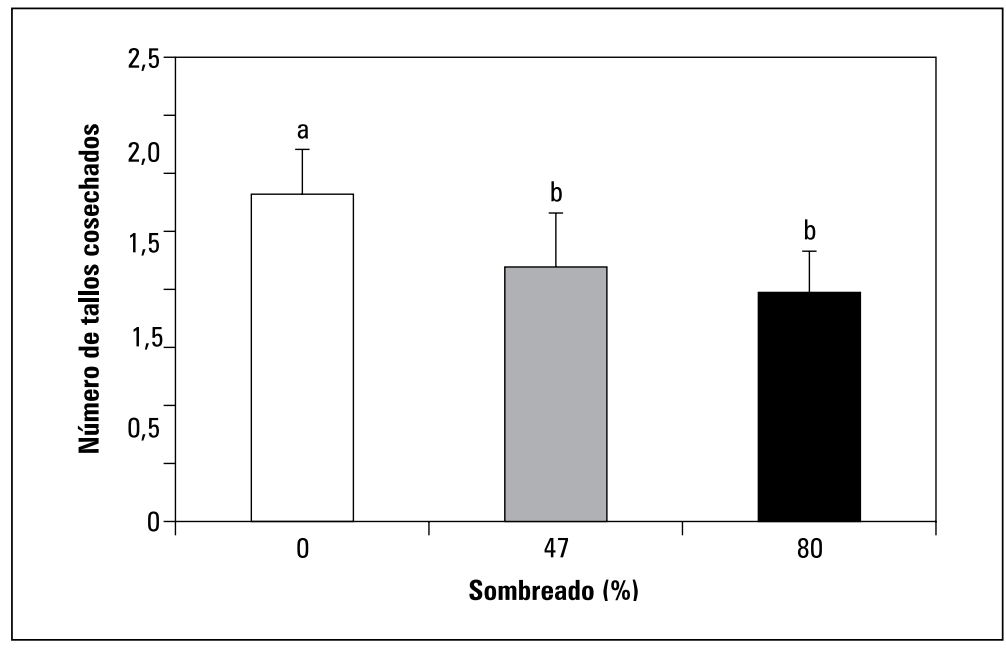

Figura 3. Efecto del sombreado a dos niveles sobre el número tallos cosechados en plantas de limonio (Limonium sp. cv. Bluestream). Promedios con letras distintas son significativamente diferentes según la prueba de Turkey $(P \leq 0,01)$. 


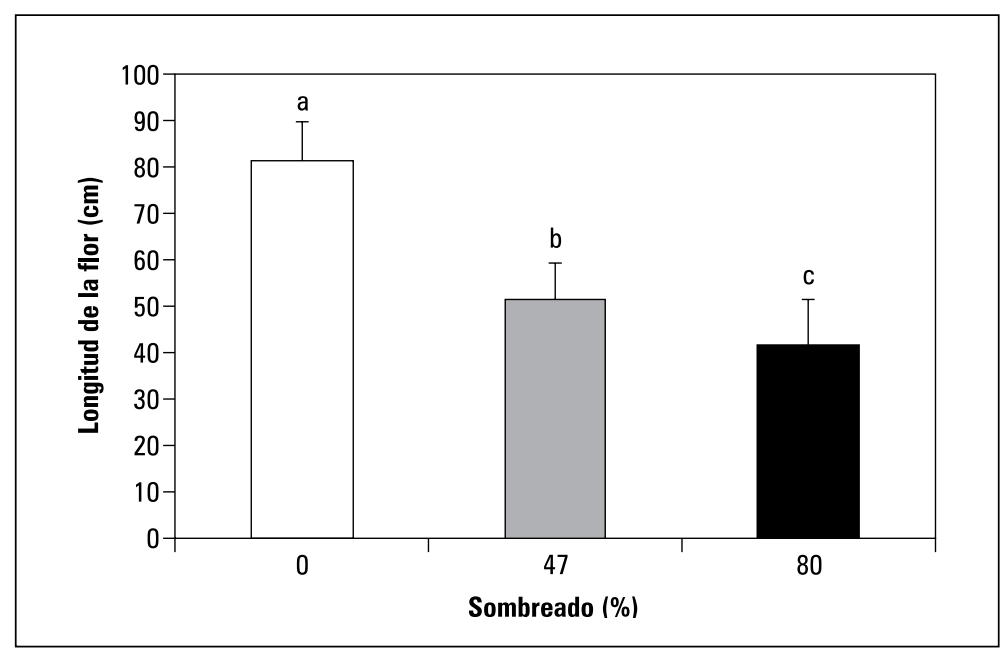

Figura 4. Efecto del sombreado a dos niveles sobre la longitud de la flor en plantas de limonio (Limonium sp. cv. Bluestream). Promedios con letras distintas son significativamente diferentes según la prueba de Turkey $(P \leq 0,01)$.

especies cultivadas, dado que de esta manera las plantas producen tallos florales de mayor longitud que cuando se cultivan a plena exposición, sin que se afecte negativamente la apariencia de la flor ni el rendimiento en número de flores (Armitage, 1991; 1993). Contrario a lo esperado, según los resultados del presente trabajo, como muestra la figura 4, la reducción en la intensidad luminosa disminuyó la longitud de los tallos florales, como consecuencia de la intolerancia de las plantas de limonio a la iluminación baja.

Resultados similares se han obtenido en otras especies vegetales para producción de flores de corte. En plantas de Liatris spicata la longitud de la espiga y el diámetro del tallo floral se redujeron al aumentar el grado de sombreamiento a las plantas lo cual afectó la firmeza de las varas. El tratamiento con $80 \%$ de sombra produjo los valores más bajos en estos dos parámetros. Si bien es cierto que se obtuvieron varas más largas con $65 \%$ de sombra, estas fueron más débiles y produjeron espigas más cortas que las plantas de tratamiento control. Esto indica que no es recomendable el uso de mallas polisombra en octubre, para las condiciones de Chile (Schiappacasse et al., 2007). De igual manera en plantas de Liatris se observó un retraso en la maduración, que los autores atri- buyeron a la reducción en la temperatura como consecuencia de la malla polisombra, que pudo retrasar el crecimiento de las plantas. (Schiappacasse et al., 2007). De igual manera, las bajas temperaturas y su efecto sobre el crecimiento y el retraso en la floración de Liatris fue observado por Espinosa et al. (1991), pero Chang et al. (2004) encontraron que diferentes variedades de Statice sp. mostraron buen comportamiento en cuanto a la producción de flores bajo condiciones de sombrío, debido a que las mallas polisombra redujeron la temperatura alrededor de las plantas cuando se presentaron temperaturas cercanas a $36^{\circ} \mathrm{C}$, lo que contribuyó a prevenir la desvernalización por el efecto de las temperaturas altas.

Acorde con los resultados obtenidos en el presente estudio, las condiciones de cultivo de limonio en la zona del ensayo no serían las más adecuadas para una aceptable producción de flores de la especie evaluada, dado que se trata de plantas muy exigentes en luminosidad, pues según reporte de Escher (1996), la producción de flores de limonio se reduce bajo condiciones de cielo nublado; razón por la cual, es posible incrementar entre 50 y $100 \%$ la producción de flor cosechada en el sur de Israel con respecto a la obtenida en la zona norte del mismo país, dado que en el sur, 
la iluminación es $15 \%$ superior a la que se registra en el norte. Bajo estas consideraciones, si solo las instalaciones del invernadero reducen la radiación incidente en $34,4 \%$, sería de esperarse que con el uso de las mallas polisombra que reducen 63,8 y 79,8\% la luminosidad, se produjeran mermas en la producción y calidad de la flor. Por tanto, esta especie se debe cultivar en zonas de alta iluminación, dada su poca adaptación a las condiciones de iluminación reducida.

\section{AGRADECIMIENTOS}

Este estudio se desarrolló con el apoyo de la Dirección de Investigaciones (DIN) de la Universidad Pedagógica y Tecnológica de Colombia, en el marco del plan de trabajo del grupo de investigación Ecofisiología Vegetal, adscrito al programa de Ingeniería Agronómica de la Facultad de Ciencias Agropecuarias.

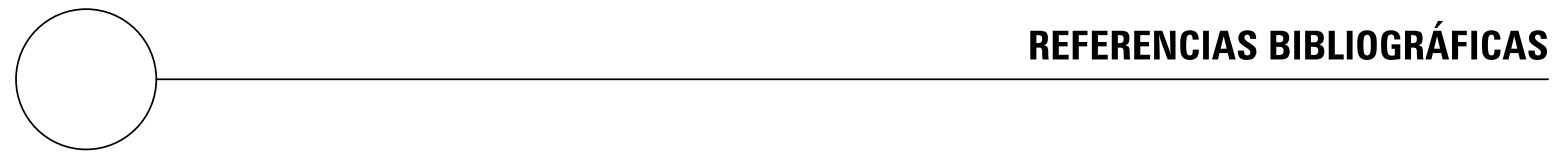

Abrams, M.C. y S.C. Mosteller. 1995. Gas exchange, leaf structure and nitrogen in contrasting tree species growing in open and understory sites during a drought. Tree Physiol. 15, 361-370.

Alexieva, V.; S. Ivanov; I. Sergiev y E. Karanov. 2003. Interaction between stresses. Bulg. J. Plant Physiol., special issue. 1-17.

Armitage, A.M. 1991. Shade affects yield and stem length of field-grown cut. flower species. HortScience 26, 1174-1176

Armitage, A.M. 1993 Specialty cut flowers. Varsity Press, Inc. Timber Press, Portland, Oregon. 372 p.

Asocolflores. 2004. Estadística. En: www.org/info/info.php; consulta: marzo de 2005.

Bazzaz F.A. y R.W. Carlson. 1982. Photosynthetic acclimation to variability in the light environment of early and late successional plants. Oecologia 54, 313-316.

Ben Haj Salah, H. y F. Tardieu. 1995. Temperature affects leaf expansion rate of maize without change in spatial distribution of cell length. Analysis of the coordination between cell division and cell expansion. Plant Physiol. 109, 861-870.

Casierra-Posada, F. 1999. Einfluß der Hagelschutznetze und des Sommerschnittes auf Apfelbäume bei unterschiedlicher Stickstoffversorgung. Tesis doctoral. Humboldt Universität zu Berlín, 104 p.

Casierra-Posada, F. y P. Lüdders. 2001. Fotosíntesis y consumo de agua en árboles de manzano 'Golden Delicious' bajo sombra. Revista Comalfi 28(1), 1-6.
Casierra-Posada, F. y P. Lüdders. 2001a. Einfluß von Sommerschnitt und N-Ernährung auf den Mineralstoffgehalt von Apfelbäumen unter Hagelschutznetz. Erwerbsobstbau 43, 106-113.

Castro-Díez P.; J. Navarro; A. Pintado; L.G. Sancho y M. Maestro. 2006. Interactive effects of shade and irrigation on the performance of seedlings of three Mediterranean Quercus species. Tree Physiol. 26, 389-400.

Chamorro, A.H.; S.L. Martínez; J.C. Fernández y T. Mosquera. 2007. Evaluación de diferentes concentraciones de algunos reguladores de crecimiento en la multiplicación y enraizamiento in vitro de Limonium var. Misty blue. Agron. Colomb. 25(1), 47-53.

Chang, Y.T.; Y.C. Wang; C.S. Chang; S.S. Wang y D.L. Lin. 2004. Effects of treat shading net to avoid devernalization of statice. pp. 59-66. En: http://book.tndais. gov.tw/RBulletin/44-4.pdf; consulta: 17 de agosto de 2007.

Dengler, N.G. 1980. Comparative histological basis of sun and shade leaf dimorphism in Helianthus annuus. Can. J. Bot. 58, 717-730.

Ebert, G. y F. Casierra. 2000. Verringert die Einnetzung grundsätlich die Assimilationsleistung von Apfelbäumen?. Erwerbsobstbau 42, 12-14.

Escher, F. 1996. Kulturbeschreibungen. pp. 414-420. En: Escher, F. (ed.). Schnittblumenkulturen. Ulmer Verlag, Stuttgart.

Espinosa, I.; W. Healy y M. Roh. 1991. The role of temperature and photoperiod on Liatris spicata Shoot development. J. Amer. Soc. Hort. Sci. 116, 27-29. 
Franklin, K.A. y G.C. Whitelam. 2005. Phytochromes and shade-avoidance responses in plants. Ann. Bot. 96, 169-175.

Givnish, T.J. 1988. Adaptation to sun and shade: A wholeplant perspective. Aust. J. Plant Physiol. 15, 63-92.

Granier, C. y F. Tardieu. 1998. Is thermal time adequate for expressing the effects of temperature on sunflower leaf development?. Plant Cell Environ. 21, 695-703.

Granier, C.; C. Massonnet ; O. Turc ; B. Muller ; K. Chenu y F. Tardieu. 2002. Individual leaf development in Arabidopsis thaliana: a stable thermal-timebased programme. Ann. Bot. 89, 595-604.

Guarnaschelli, A.B.; G. Gutiérrez; A. Battaglia; P. Pathauer y J.H. Lemcoff. 2004. Influence of shading on physiology, morphology and growth of Eucalyptus seedlings. Conferencia Internacional IUFRO Eucalyptus in a Changing World. Aveiro, Portugal. 1115/10/2004 (en medio magnético).

King, D.A. 1994. Influence of light level on the growth and morphology of samplings in a Panamanian forest. Amer. J. Bot. 81, 948-957.

Kitajima, K. 1994. Relative importance of photosynthetic traits and allocation patterns as correlates of seedling shade tolerance of 13 tropical trees. Oecologia 98, 419-428.

Kobe, R.K. 1997. Carbohydrate allocation to storage as basis of interspecific variation in sampling survivorship and growth. Oikos 80, 226-233

Kozuka, T;; G. Horiguchi; G.T. Kim; M. Ohgishi T. Sakai y H. Tsukaya. 2005. The different growth responses of the Arabidopsis thaliana leaf blade and the petiole during shade avoidance are regulated by photoreceptors and sugar. Plant Cell Physiol. 46, 213-223.

Kristoffersen, T. 1963. Interactions of photoperiod and temperature in growth and development of young tomato plants. Physiol. Plant. Suppl. 1, 1-98.

Lafarge, T; M. de Raïssac y F. Tardieu. 1998. Elongation rate of sorghum leaves has a common response to meristem temperature in diverse African and European conditions. Field Crop Res. 58, 69-79.

Lambers, H.; F.S. Chapin y T.J. Pons. 1998. Plant Physiological Ecology. Springer-Verlag, New York. 540 p.

Lambers, H. y H. Poorter. 1992. Inherent variation in growth rate between higher plants: A search for physiological causes and ecological consequences. Adv. Ecol. Res. 23, 187-261.

Lee, D.W.; S.F. Oberbauer; P. Johnson; B. Krishnapilay; M. Mansor; H. Mohamad y K.S. Yap. 2000. Effects of irradiance and spectral quality on leaf structure and function in seedlings of two Southeast Asian Hopea (Dipterocarpaceae) species. Amer. J. Bot. 87, 447-455.

Lichtenthaler, H.K. 1983. Differences in morphology and chemical composition of leaves grown at different light intensities and qualities. pp. 201-221. En: Baker N.R., W.J. Davies y C.K. Ong (eds.). Control of leaf growth. University Press, Cambridge.

Lusk, C.H. 2002. Leaf area accumulation helps juvenile evergreen trees tolerate shade in temperate rainforest. Oecologia 132, 188-196.

Lusk, C.H. 2004 Adaptación a la sombra en especies arbóreas siempre/verdes. pp. 235-247. En: Cabrera, H.M. (ed.). Fisiología ecológica en plantas: Mecanismos y respuestas a estrés en los ecosistemas. Ediciones Universitarias de Valparaíso, Pontificia Universidad Catolica de Valparaíso, Chile.

Lusk, C.H. y O. Contreras. 1999. Foliage area and crown nitrogen turnover in temperate rainforest juvenile trees of differing shade tolerance. J. Ecol. 87, 973-983.

Niimets, Ü. 1998. Growth of young trees of Acer platanoides and Quercus rubor along a gap-understory continuum: Interactionships between allometry, biomass partitioning, nitrogen and shade-tolerance. Int. J. Plant Sci. 159, 318-330.

Reich, P.B. 1993. Reconciling apparent discrepancies among studies relating life span, structure and function of leaves in contrasting plant life form and climates: "The blind men and the elephant retold". Funct. Ecol. 7, 721-725.

Schiappacasse, F.; G. Carrasco S. y F. Carrasco C. 2007 Efecto de cuatro niveles de sombreamiento sobre la calidad de vara floral y cormo de liatris (Liatris spicata). Agricultura Técnica 67(1), 100-104.

Shifriss, C; M. Pilowsky y B. Aloni. 1994. Variation in flower abscission of peppers under stress shading conditions. Euphytica 78(1-2), 133-136.

Schlichting, C.D. 1986. The evolution of phenotypic plasticity in plants. Annu. Rev. Ecol. Syst. 17, 677-693.

Schmalhausen, I.I. 1949. Factors of evolution: The theory of stabilizing selection. Chicago University Press. $483 \mathrm{p}$.

Schnyder, H. y C.J. Nelson. 1988. Diurnal distribution of tall fescue leaf blades. I. Spatial distribution of growth, deposition of water, and assimilate import in the elongation zone. Plant Physiol. 86, 10701076.

Tognetti R.; M. Michelozzi y M. Borghetti. 1994. Responses to light of shade-grown beech seedlings subjected to different watering regimes. Tree Physiol. 14, 751-758. 
Veneklaas, E.J. y L. Poorter. 1998. Growth and carbon partitioning of tropical tree seedlings in contrasting light environments. En: Lambers, H.; M.N.I. van Vuuren (eds.). Inherent variation in plant growth. Physiological mechanisms and ecological consequences. Backhuys Publishers, Leiden, Holanda. 600 p.

Volenec,J.J.yC.J.Nelson.1982.Diurnalleafelongationofcontrasting tall fescue genotypes. Crop Sci. 22, 531-535.

Walters, M.B. y P.B. Reich. 1999. Research review lowlight carbon balance and shade tolerance in the seedling of woody plants: Do winter deciduous and broadleaved evergreen species differ?. New Phytol. $143,143-154$.
Williams, K.; C.B. Field y H.A. Mooney. 1989. Relationships among leaf construction cost, leaf longevity and light environment in rain-forest plants of the genus Piper. The American Naturalist 133, 198211.

Wilson G.L. 1966. Studies on the expansion of the leaf surface. V. Cell division and expansion in a developing leaf as influenced by light and upper leaves. J. Exp. Bot. 17, 440-451.

Yano, S. y I. Terashima. 2004. Developmental process of sun and shade leaves in Chenopodium album $\mathrm{L}$. Plant Cell Environ. 27, 781-793. 